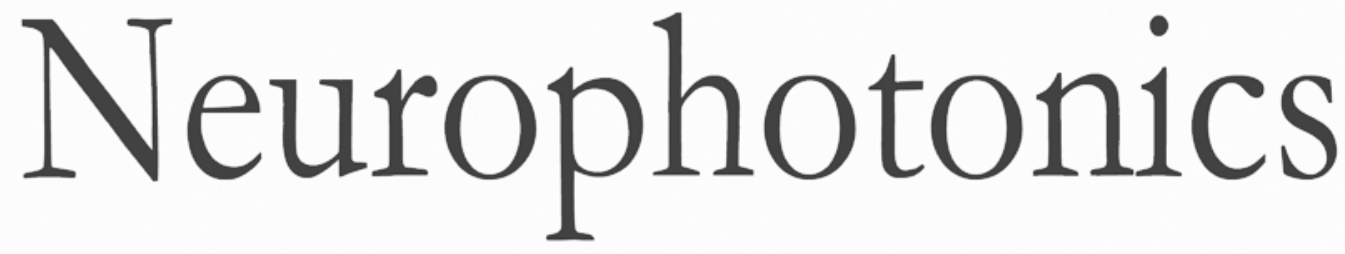

\title{
Design of multichannel functional near-infrared spectroscopy system with application to propofol and sevoflurane anesthesia monitoring
}

Zhenhu Liang Yue $\mathrm{Gu}$

Xuejing Duan

Lei Cheng

Shujuan Liang

Yunjie Tong

Xiaoli Li 


\title{
Design of multichannel functional near-infrared spectroscopy system with application to propofol and sevoflurane anesthesia monitoring
}

\author{
Zhenhu Liang, ${ }^{a}$ Yue Gu, ${ }^{a}$ Xuejing Duan, ${ }^{a}$ Lei Cheng, ${ }^{a}$ Shujuan Liang, ${ }^{b}$ Yunjie Tong, ${ }^{c}$ and Xiaoli Li ${ }^{\mathrm{d}, e, *}$ \\ aYanshan University, Institute of Electrical Engineering, No. 438 Hebei Street, Haigang District, Qinhuangdao 066004, China \\ ${ }^{b}$ Department of Anesthesia, No. 1 Hospital of Qinhuangdao, No. 258 Wenhua Street, Haigang District, Qinhuangdao 066004, China \\ 'McLean Hospital, McLean Imaging Center, 115 Mill Street, Belmont, Massachusetts 02478, United States \\ ${ }^{\mathrm{d} B e i j i n g ~ N o r m a l ~ U n i v e r s i t y, ~ S t a t e ~ K e y ~ L a b o r a t o r y ~ o f ~ C o g n i t i v e ~ N e u r o s c i e n c e ~ a n d ~ L e a r n i n g ~ \& ~ I D G / M c G o v e r n ~ I n s t i t u t e ~ f o r ~ B r a i n ~ R e s e a r c h, ~ N o . ~} 19$ \\ Xinjiekou Wai Street, Haidian District, Beijing 100875, China \\ ${ }^{\mathrm{e} B e i j i n g ~ N o r m a l ~ U n i v e r s i t y, ~ C e n t e r ~ f o r ~ C o l l a b o r a t i o n ~ a n d ~ I n n o v a t i o n ~ i n ~ B r a i n ~ a n d ~ L e a r n i n g ~ S c i e n c e s, ~ N o . ~} 19$ Xinjiekou Wai Street, Haidian District, \\ Beijing 100875, China
}

\begin{abstract}
Monitoring the changes of cerebral hemodynamics and the state of consciousness during general anesthesia (GA) is clinically important. There is a great need for developing advanced detectors to investigate the physiological processes of the brain during GA. We developed a multichanneled, functional near-infrared spectroscopy (fNIRS) system device and applied it to GA operation monitoring. The cerebral hemodynamic data from the forehead of 11 patients undergoing propofol and sevoflurane anesthesia were analyzed. The concentration changes of oxygenated hemoglobin, deoxygenated hemoglobin, total hemoglobin, and cerebral tissue heart rate were determined from the raw optical information based on the discrete stationary wavelet transform. This custom-made device provides an easy-to-build solution for continuous wave-fNIRS system, with customized specifications. The developed device has a potential value in cerebral monitoring in clinical settings. ๑ 2016 Society of Photo-Optical Instrumentation Engineers (SPIE) [DOI: 10.1117/1.NPh.3.4.045001]
\end{abstract}

Keywords: cerebral hemodynamic; general anesthesia; functional near-infrared spectroscopy; discrete stationary wavelet transform. Paper 16035RRR received Jun. 17, 2016; accepted for publication Sep. 12, 2016; published online Oct. 5, 2016.

\section{Introduction}

Understanding the neurophysiological mechanisms of anesthetic drug effects on the brain is a hot topic in recent years. ${ }^{1}$ It is known that an underdose of anesthetics may cause intraoperative awareness or postoperative recall whereas an overdose may lead to recovery delay or cardiopulmonary complication risks. ${ }^{2}$ Although there is a variety of intraoperative monitoring equipment, such as multiparameter physiological monitors based on electroencephalogram (EEG), electrocardiogram, blood pressure, pulse oximetry, and so on; patients are still at risk of an anesthetic overdose or underdose within clinical practice. ${ }^{3}$ Providing a safe and reliable intraoperative monitoring system remains a challenging issue. ${ }^{4}$

Several commercially available, EEG-based depth of anesthesia (DoA) monitors, such as the bispectral index (BIS) monitor (Medtronic, Dublin, Ireland) ${ }^{5}$ and M-entropy (GE Healthcare, Helsinki, Finland), ${ }^{6}$ have been developed in recent decades. ${ }^{5,7,8}$ The advantages of EEG-based monitors include noninvasiveness, high portability, high time resolution, and direct assessment of the brain states under general anesthesia (GA). ${ }^{9}$ However, the routine use of EEG monitors was estimated not to exceed $1.8 \% .^{10}$ The reasons for the low usage of these devices included: (1) the poor performance in the presence of opioids, ${ }^{11}$ (2) the lack of direct information provided to guide the delivery of anesthetics; ${ }^{12}$ and (3) the lack of assessment of cerebral oxygen saturation, which is also important in intraoperative and postoperative intensive care. ${ }^{13,14}$ These studies indicate that EEG measurement alone is not adequate for DoA monitoring.
Functional near-infrared spectroscopy (fNIRS) is a neuroimaging tool that monitors cerebral metabolic activity by measuring the absorption of near-infrared (NIR) light between 650 and $950 \mathrm{~nm}$ noninvasively. ${ }^{15}$ As the absorption spectra of deoxyhemoglobin $(\mathrm{Hb})$ and oxyhemoglobin $\left(\mathrm{HbO}_{2}\right)$ are distinct in this region, it is possible to determine the concentration changes of $\mathrm{HbO}_{2}$ and $\mathrm{Hb}$ individually by using the infrared lights of two distinct wavelengths. The two wavelengths of NIR light for measuring $\mathrm{HbO}_{2}$ and $\mathrm{Hb}$ are usually selected on both sides of the isosbestic point $(805 \mathrm{~nm})$, one above and the other below this point (e.g., 730 and $850 \mathrm{~nm}$ ). fNIRS supplies a safe, nonionizing radiative, noninvasive, and cost-effective measurement of cerebral hemodynamic changes in cerebral cortex in comparison with positron emission tomography (PET) and functional magnetic resonance imaging (fMRI). ${ }^{16,17}$ Over the years, this technique has evolved into a versatile medical diagnostic modality and has been used to investigate many brain functions. ${ }^{18-23}$

In general, there are three types of fNIRS systems: continuous wave $(\mathrm{CW})$ modality, frequency-domain (FD) modality, and time-domain modality. ${ }^{24}$ As the CW-based systems offer the advantages of relatively low cost, high adaptability, and portability, most of commercially available NIRS systems are CWbased. ${ }^{25,26}$ The food and drug administration has approved four cerebral oximeter devices for clinical monitoring: the CerOx (Ornim, Foxboro, Massachusetts), Equanox (Nonin Medical, Plymouth, Minnesota), FORE-SIGHT (CAS Medical Systems, Branford, Connecticut), and INVOS (Covidien, Dublin, Ireland). ${ }^{27}$ 
However, the oximetry of FORE-SIGHT and INVOS can only supply physiological changes rather than changes with cognitive activity. ${ }^{28}$ Further, quantities measured by these devices may be altered by many factors, such as cardiac output, hypo/hypercapnia, temperature, and local blood flow. Additionally, indices must be interpreted in the context of available clinical information if they are supposed to be used to track anesthesia process. Stable systems and robust indicators are still needed.

The ability of fNIRS to detect the major events of cerebral ischemia is well established, ${ }^{29}$ however, the relation of cerebral ischemia and the progress of anesthesia is not clear. ${ }^{30}$ Previous studies found that an $\mathrm{HbO}_{2}$ concentration increased 3 min after propofol induction. ${ }^{31}$ Fassoulaki et al. found that the regional cerebral oxygenation did not differ between sevoflurane and desflurane as BIS values were maintained within the range of 40 to 50 or 20 to $30 .{ }^{32}$ Leon-Dominguez et al. found that the $\mathrm{Hb}$ in the prefrontal cortex (PFC) increased during the induction phase with propofol and remifentanil induction in colopoctology surgery ${ }^{33}$ while the Hb levels of different channels were altered during the emergence phase. Curtin et al. used the same device to measure the propofol effect during an outpatient elective colonoscopy. The results showed that the relative $\mathrm{Hb}$ value was decreased during the induction and relative $\mathrm{HbO}_{2}$ indices were significantly different at different propofol dosages. ${ }^{21}$ Further, the effect of inhalation of anesthetic sevoflurane on cerebral oxygenation was studied. It was found that oxygen saturation is sensitive to inspire oxygen and subject position. ${ }^{34,35}$ Hernandez-Meza et al. ${ }^{28}$ provided a thorough review of the use of fNIRS in the fields of anesthesia and sedation monitoring. It is clear that there are still many problems to be solved in using fNIRS to monitor brain states under GA in clinic.

Since NIRS measurements are known to be sensitive to absorption changes in the superficial layers in functional studies, a significant source of noise in optical studies is due to physiological signals in these superficial layers. These signals include systemic physiological hemodynamic fluctuations, such as cardiac pulsation, respiratory signals, and blood pressure changes, which are presented in the scalp and underlying cerebral tissue. $^{36-38}$ Although these physiological waveforms are considered to be the artifact in estimating the cerebral metabolic activity, there are also some other research groups that use this information to explore the vascular physiology in healthy subjects and stroke patients. ${ }^{39,40}$ Hemmerling et al. developed an objective score (analgoscore) of intraoperative pain based on the mean arterial pressure and heart rate (HR) for close loop control of remifentanil. ${ }^{41,42}$ Cardiac pulsation (around $1 \mathrm{~Hz}$ ) is one of the predominant physiological processes in fNIRS. ${ }^{43}$ Perdue et al. had proved that the HR derived from the head in fNIRS were highly correlated with the HR calculated from the electrocardiography. ${ }^{44}$ This motivated us to consider if the cardiac pulsation noise in NIRS could be used as an effective signal in DoA monitoring. In this study, in order to make a distinction in the HR from the peripheral, we denoted the HR parameter of the cerebral tissue as cerebral tissue heart rate (CTHR).

The primary purpose of this paper is to introduce a homemade, multichanneled, fNIRS system for measuring frontal hemodynamics and to evaluate its performance on DoA monitoring. This paper is organized as follows: the next section addresses the fundamental principles of fNIRS, the sourcedetector probe schematic, and the hardware and software architecture of this system. Section 3 describes the experimental evaluation of the system. In Sec. 4, fNIRS data acquisition in clinic, preprocessing and cerebral hemodynamic information and CTHR extraction are discussed in detail. Section 5 gives a detailed illustration of the hemodynamic information derived under GA. Finally, a discussion and conclusion are given in Sec. 6.

\section{Frequency Division Multiplexing Functional Near-Infrared Spectroscopy System}

\subsection{Principles of Frequency Division Multiplexing Functional Near-Infrared Spectroscopy}

The typical CW-based NIRS system emits constant light into the tissue, and an avalanche photo diode or photomultiplier tube is used to measure the amplitude of the remnant light that is reflected back. This kind of system is susceptible to the interference with the ambient light. ${ }^{26,45}$ The frequency division multiplexing method adopts the sinusoidal wave to modulate the source. Different sources are paired with different modulation frequencies, which are usually selected from 1 to $10 \mathrm{kHz}$ (similar to CW5 imaging system from TechEn, Inc., Milford, Massachusetts). The detected signals are demodulated to extract the corresponding cerebral hemodynamic information. The benefits of this method include: (1) immunity to ambient light and (2) simultaneous multichannel detection.

\subsection{Multichannel Probe and Source-Detector Schematic of This Device}

In order to detect the hemodynamic changes of the PFC, we designed a simple, multichanneled probe, which can be used in the operating room. The source-detector schematic is shown in Fig. 1, with three light emitting diodes (LEDs), and eight Si-photodiodes (PD) forming 12 source-detector channels. The horizontal distance between the nearest two detectors is $4.8 \mathrm{~cm}$ and the vertical distance is $4 \mathrm{~cm}$, so the distance between the nearest detector and source is about $3 \mathrm{~cm}$. The NIR light source used in the system is L760/850-04A (EXPITEX Inc. Kyoto, Japan), which emits two wavelengths of light (760 and $850 \mathrm{~nm}$ ). The re-emerging NIR light is collected and converted to an electrical signal by Si-PD. The Si-PD S2386-44K (Hamamatsu, Inc. Hamamatsu, Japan) has the features of high linearity, high sensitivity, and short rise time, which make it suitable for high-frequency modulation and weak light conversion.

\subsection{Hardware and Software Architecture}

The hardware platform is composed of three modules: a digital-to-analog converter (DAC) and LED-driver module, a programmable gain amplifier (PGA) and analog-to-digital

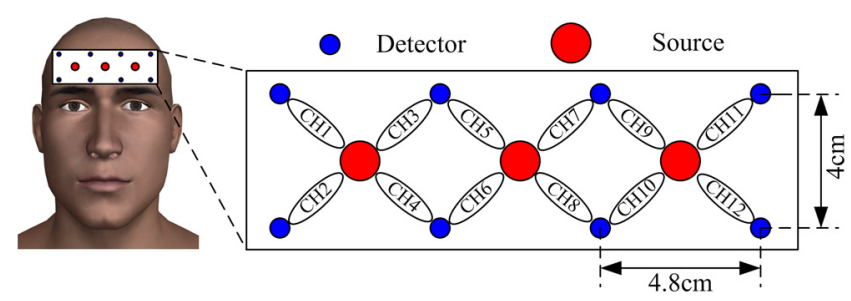

Fig. 1 The source-detector schematic of the multichanneled probe. It consists of three LED sources and eight Si-PD detectors forming 12 channels ( $\mathrm{CH} 1-\mathrm{CH} 12)$. The horizontal distance between the nearest two detectors is $4.8 \mathrm{~cm}$ and the vertical distance is $4 \mathrm{~cm}$. 
converter (ADC) module, and a field programmable gate array (FPGA) controller and universal serial bus (USB) interface module. The functions of the LabVIEW software system consist of USB data reading, signal demodulation, hemodynamic information calculation based on the modified Beer-Lambert law (MBLL), signal display, and storage. The architecture of the system is shown in Fig. 2, and the details of each part are described in the following paragraphs.

The DAC (PCM1690, Texas Instruments Inc., Texas) generates sinusoidal carrier signals ranging from 1 to $7 \mathrm{kHz}$ with an interval of $1 \mathrm{kHz}$ (under the control of the FPGA). The low pass filter eliminates the high-frequency noise mixed within the carrier signal. The driving circuit of light source converts the voltage signal to current signal (VI) to drive the LEDs. The average output power of 760 and $850 \mathrm{~nm}$ light sources is about 16 and $18 \mathrm{~mW}$, respectively. The detected light is magnified by a PGA (PGA204, Texas Instruments Inc., Texas) and then digitized by ADC (PCM4201, Texas Instruments Inc., Texas) with the sampling rate of $100 \mathrm{kS} / \mathrm{s}$ and 24-bit resolution. In order to remove the effect of aliasing in digitized signals, the sampling rate is commonly set to be $\sim 5$ times the maximum frequency. ${ }^{46}$ Also, considering the future upgrade of the system (expansion in the light sources), we adopted $100 \mathrm{kHz}$ as a more redundant design. The time sequence of each ADC channel is controlled by the FPGA concurrently. Data transfer and power supply are realized by a USB 2.0 interface connected to a notebook computer through the Virtual Instrument Software Architecture. The software architecture of this system is shown in Fig. 2 within the dotted box.

The wearable headset is made of soft materials to ensure comfortable wear. The custom-designed data acquisition board and source driving circuit are placed in a $125 \mathrm{~mm} \times$ $110 \mathrm{~mm} \times 50 \mathrm{~mm}$ aluminum enclosure. The whole system is suitable to be placed next to the operating table. The probe [Fig. 3(a)], the disassembled prototype [Fig. 3(b)], the instrument appearance [Fig. 3(c)], and the software interface [Fig. 3(d)] of this device are shown in Fig. 3.

\section{Experimental Evaluation}

The effectiveness of the custom-made fNIRS system was verified by performing a Valsalva maneuver task, ${ }^{47}$ which contained five blocks and each block included $30 \mathrm{~s}$ breath-hold and $120 \mathrm{~s}$ rest period. Data were recorded by our system and a commercial fNIRS system NIRScout (NIRx Medical Technologies, LLC.) simultaneously. The emitters and detectors of the two systems were placed side-by-side on the prefrontal area around the FP1 position according to the 10-20 system. The experimental paradigm and sessions were designed using the E-prime software (Psychology Software Tools). The trigger signals from the Eprime were sent to the custom-made system and the NIRScout via serial and parallel port, respectively, to synchronize all the devices. The instruction of the Valsalva maneuver task includes taking a deep breath, closing the mouth, pinching the nose, and attempting to expire air, as well as keeping the cheek muscles tight.

Data were analyzed later in MATLAB ${ }^{\circledR}$. First, the signals of our custom-made fNIRS system were downsampled to $10 \mathrm{~Hz}$. Then, the $\mathrm{HbO}_{2}$ and $\mathrm{Hb}$ concentration changes were calculated based on MBLL. These hemodynamic signals were expressed as the product of the relative change of hemoglobin concentration and the effective optical path length $(\mathrm{mM} \times \mathrm{mm})$. Finally, after a band-pass filtering ( 0.004 to $2 \mathrm{~Hz}$ ), the $\mathrm{HbO}_{2}$, and $\mathrm{Hb}$ concentration changes can be visually inspected, as shown in Fig 4. Figures 4(a) and 4(b) are the concentration changes in $\mathrm{HbO}_{2}$ and $\mathrm{Hb}$. The yellow shaded area indicates the task period of $30 \mathrm{~s}$ breath holding. It could be highlighted that both $\mathrm{HbO}_{2}$ and $\mathrm{Hb}$ derived from the custom-made (in red) system match those from NIRScout (in blue). The correlation coefficients (corrcoef.m) of $\mathrm{HbO}_{2}$ is $0.82(p<0.001)$ and $\mathrm{Hb}$ is $0.91(p<0.001)$.

\section{Clinical Data Acquisition}

\subsection{Subjects and Data Acquisition}

This device was tested to collect cerebral hemodynamic data under GA in the No.1 Hospital of Qinhuangdao. The study

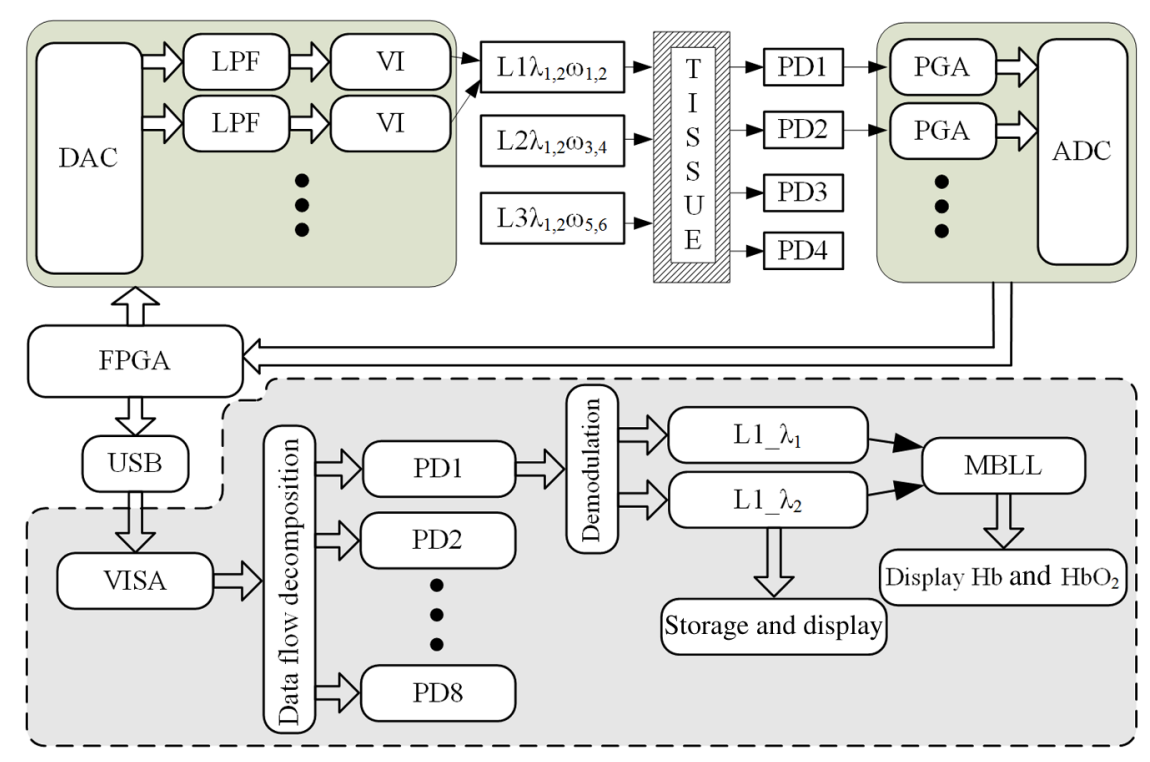

Fig. 2 The hardware and software architecture of the multichanneled fNIRS system. The hardware platform consists of a DAC and LED-driver module, a PGA and ADC module, and an FPGA controller and USB interface module. The function of the LabVIEW software system consists of USB data reading, data flow decomposition, signal demodulation, cerebral hemodynamic information calculated based on the MBLL, signal display, and storage. 
(a)

(b)

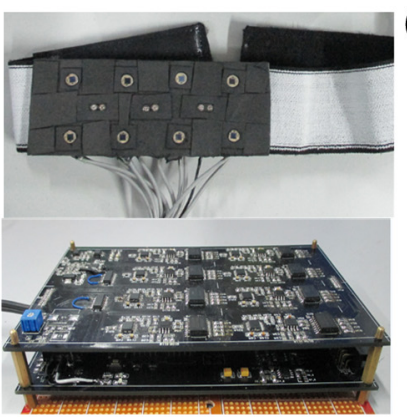

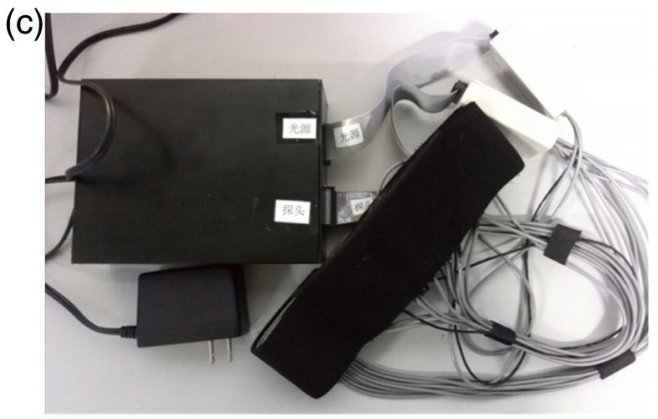

(d)

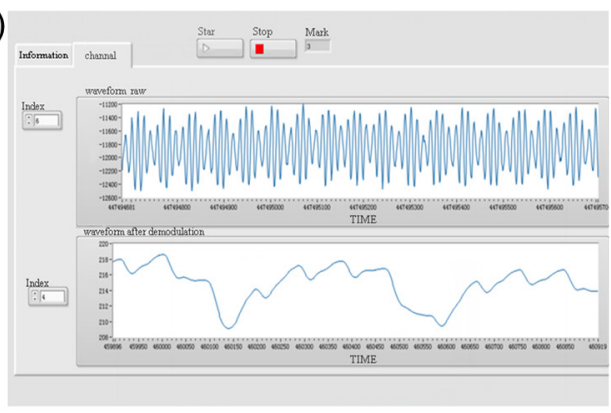

Fig. 3 The display of the multichanneled fNIRS system. (a) The wearable headset, (b) the disassembled prototype of the hardware motherboard, (c) the device appearance with the size of $125 \mathrm{~mm} \times$ $110 \mathrm{~mm} \times 50 \mathrm{~mm}$, and (d) the software interface developed based on Labview.
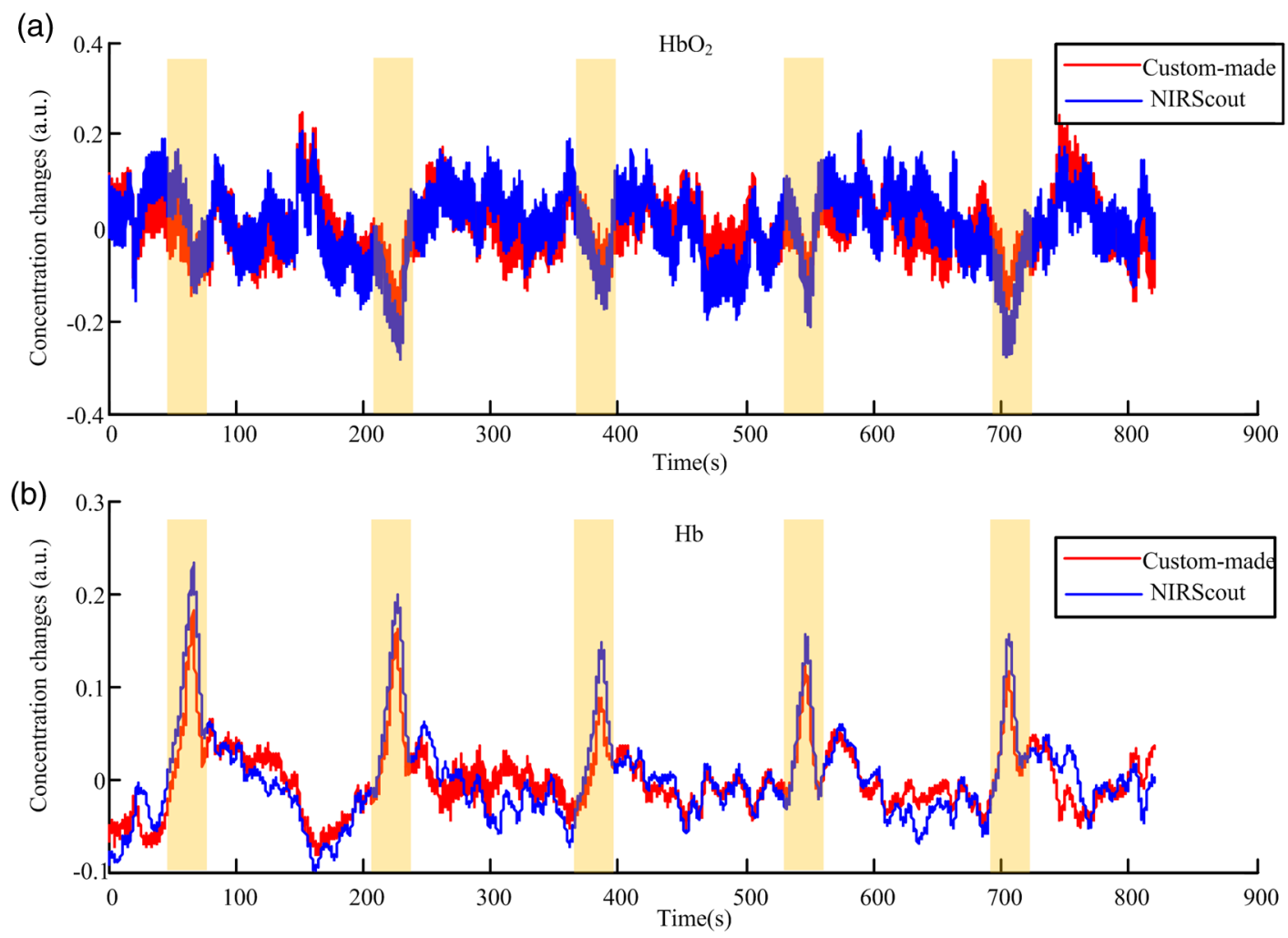

Fig. 4 Comparison of the time course of (a) $\mathrm{HbO}_{2}$ and (b) $\mathrm{Hb}$ acquired from NIRScout and a custommade device during Valsalva maneuver task.

protocol, recruitment methods, and informed consent statements were approved by the No.1 Hospital of Qinhuangdao's ethic committee. Eleven patients ( 7 males and 4 females) undergoing laparoscopic surgery were enrolled in this study. Patients were in supine position before the start of the surgery. For the surgical requirement, there were pitch angle changes during the operation in trendelenburg position. All patients were ASA (American Society of Anesthesiologists) physical status I or II. The patients, aged 18 to 62 years, had no history of seizures, head injury, chronic headaches, or neurological dysfunction and all gave written informed consent.

Anesthetics were induced intravenously with $1.5 \mathrm{mg} / \mathrm{kg}$ of propofol, $0.03 \mathrm{mg} / \mathrm{kg}$ of midazolam, $0.15 \mathrm{mg} / \mathrm{kg}$ of cisatracurium, and $4 \mu \mathrm{g} / \mathrm{kg}$ of fentanyl. The patients were preoxygenated with endotracheal intubation to satisfy oxygen demand during operation. Inhalational anesthetic (sevoflurane, $1 \%$ to $2 \%$ ) along with intravenous anesthetic (remifentanil 0.5 to $1 \mathrm{mg} / \mathrm{h}$, and propofol 150 to $300 \mathrm{mg} / \mathrm{h}$ ) were used to maintain the anesthesia state based on the patient body weight, sex, age, and patient's state. Cisatracurium was injected intermittently. The drug concentration was adjusted based on physiological parameters and the anesthesiologist's judgment on the patient's state. Loss of consciousness (LOC) was determined by the loss of response to a verbal command repeated every $5 \mathrm{~s}$ by the anesthesiologist. The "induction time" (the time point of starting induction), "operation time" (the time point of starting the surgical procedure), "anesthesia stop time" (the time point of anesthesia cessation), and "command time" (the time that the patient first responds to a verbal command during recovery) of all the patients were recorded and shown in Table 1. The awake 
Table 1 The event times for each patient (in seconds).

\begin{tabular}{lcccc}
\hline Subjects & $\begin{array}{c}\text { Induction } \\
\text { time }\end{array}$ & $\begin{array}{c}\text { Operation } \\
\text { time }\end{array}$ & $\begin{array}{c}\text { Anesthesia } \\
\text { stop time }\end{array}$ & $\begin{array}{c}\text { Command } \\
\text { time }\end{array}$ \\
\hline$\# 1$ & 230 & 1355 & 4103 & 7066 \\
$\# 2$ & 180 & 1160 & 3847 & 4868 \\
$\# 3$ & 78 & 1280 & 5947 & 6800 \\
$\# 4$ & 35 & 962 & 2355 & 4087 \\
$\# 5$ & 1050 & 2410 & $*$ & 10,500 \\
$\# 6$ & 297 & 3396 & 6965 & 7997 \\
$\# 7$ & 820 & 2186 & 5693 & 5983 \\
$\# 8$ & 686 & $*$ & 4806 & 5126 \\
$\# 9$ & 486 & 2648 & 8640 & 10860 \\
$\# 10$ & $*$ & $*$ & 6349 & 7722 \\
$\# 11$ & 99 & 2618 & 8640 & 10860 \\
\hline
\end{tabular}

Note: Induction time $=$ the time point of the start of the induction phase.

Operation time $=$ the time point of starting the surgical procedure. Anesthesia stop time $=$ the time point of anesthesia cessation.

Command time $=$ the time point corresponding to the subject's correct response to a verbal command.

${ }^{*}$ The time point is not recorded. state is the period before the "induction time." The time course between "operation time" and "anesthetic stop time" is considered as the moderate anesthesia state and the period after the "anesthetic stop time" is considered as the recovery state. The recovery of consciousness (ROC) is defined as the point when the patient could follow a verbal command.

Electrocardiographic, HR, and pulse oximetry were monitored for safety using an iPM9800 Patient Monitor (Mindray Co., Ltd. Shenzhen, China) during intraoperation. Our multichannel fNIRS system was used to collect optical signals, which were later converted to $\mathrm{HbO}_{2}$ and $\mathrm{Hb}$ signals. The wearable headset that covers the PFC was fixed on the forehead. The schematic diagram of the position of sources and detectors is shown in Fig. 1. All the data detected in the operation were recorded and stored on a laptop for off-line analysis.

\subsection{Data Preprocessing}

The collected optical data were first downsampled to $10 \mathrm{~Hz}$. Figures 5(a) and 5(c) show an epoch of the downsampled, raw optical signal of 760 and $850 \mathrm{~nm}$ from one typical patient during the operation process, respectively. Figures 5(b) and 5(d) are the corresponding log spectrum of Figs. 5(a) and 5(c), respectively. The upper portion of the figures is an enlarged section. The enlarged waveform clearly shows the cardiac pulsation, respiration cycle, and blood pressure (Mayer wave) oscillations. A motion artifact can also be found around the time point of $260 \mathrm{~s}$, where the signal intensity has a large perturbation for the probes shift.
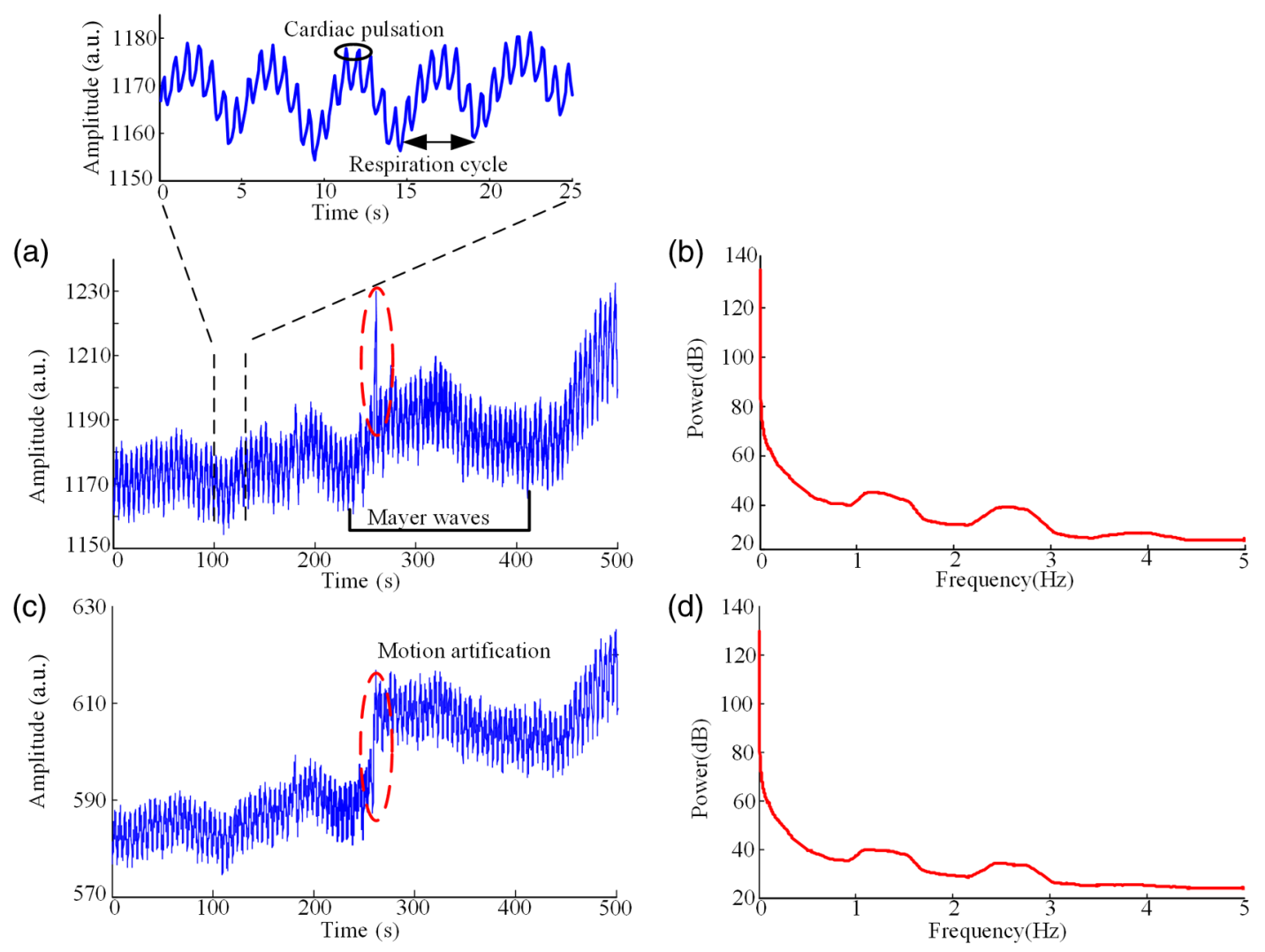

Fig. 5 The raw optical signals of 760 and $850 \mathrm{~nm}$ recorded from one channel. (a) and (c) The optical signal of 760- and 850-nm wavelength, respectively. The signals were downsampled to $10 \mathrm{~Hz}$. (b) and (d) The power spectra of optical signal of 760- and 850-nm wavelength, respectively. 


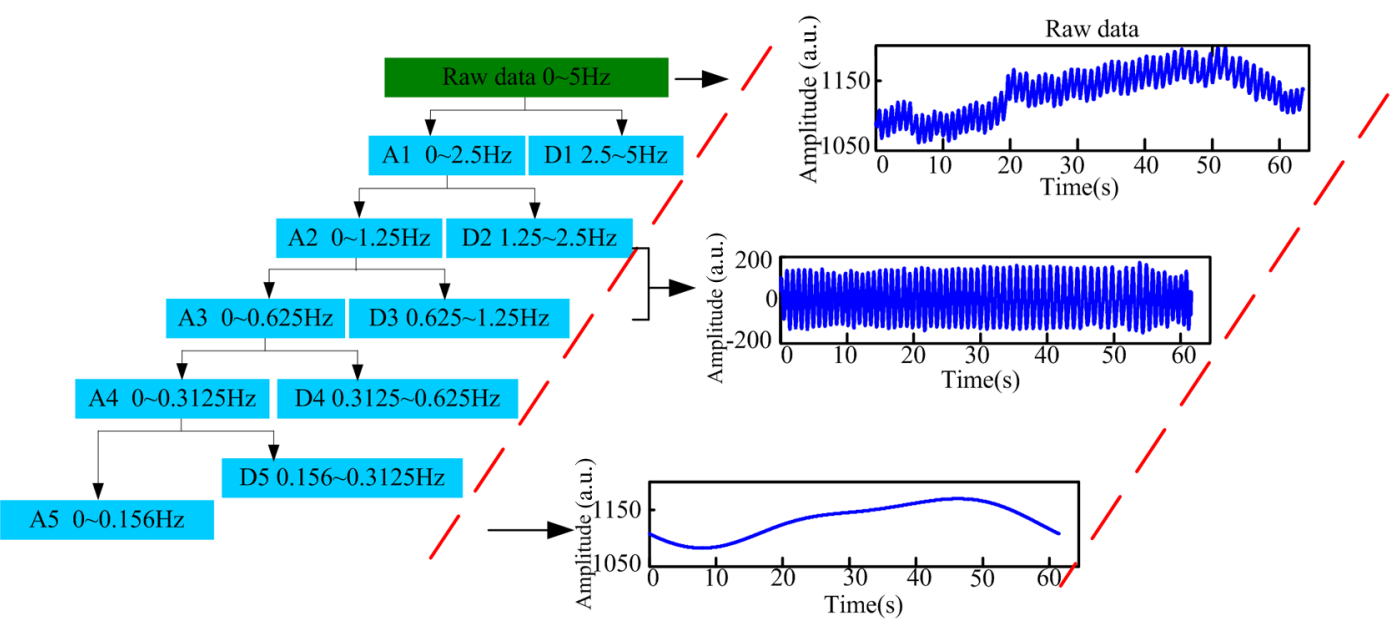

Fig. 6 The decomposition schematic diagram of a DSWT to the raw optical signals. The hemodynamic signal component localized in the approximation component of level $6(0$ to $0.156 \mathrm{~Hz})$. The detail components of D2 and D3 are used for CTHR calculation.

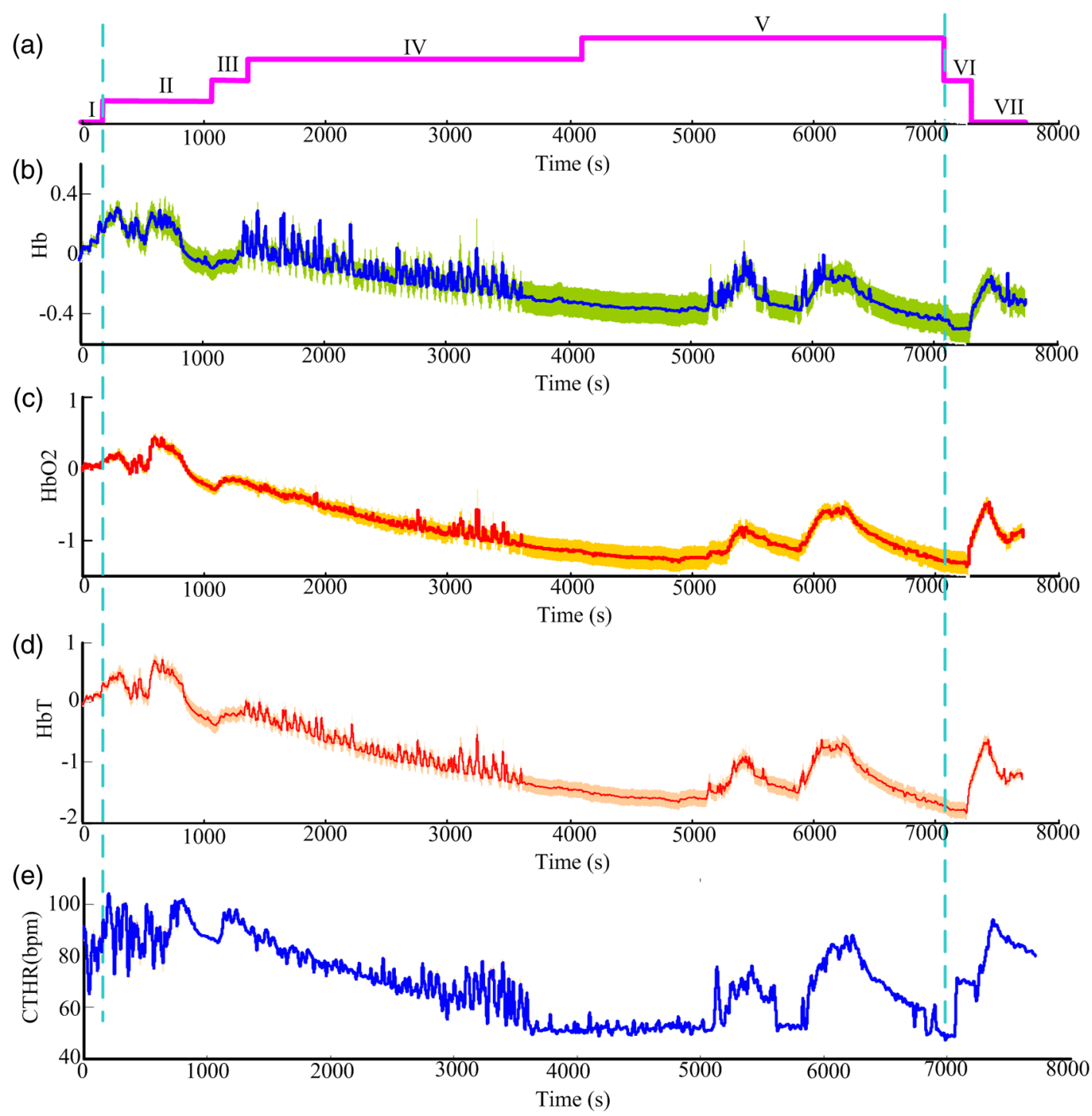

Fig. 7 The averaged cerebral hemodynamic changes of all channels for a single patient as a function of time. (a) The detailed anesthesia procedure for one patient. (I) Awake, (II) intravenous induction, (III) sevoflurane inhalation, LOC, (IV) surgery started and maintenance of the anesthesia state, (V) surgical accepted, anesthesia stopped, (VI) recovery, and (VII) consciousness. (b)-(e) The averaged concentration changes of $\mathrm{HbO}_{2}$, $\mathrm{Hb}, \mathrm{HbT}$, and CTHR of all channels as a function of time during the entire surgery. The dark solid line is the mean value of the 12 channels and the light shaded area indicates the standard deviation of all the channels. 
These studies have shown that the hemodynamic signal under GA may contain low frequency components and that simple high-pass filtering is not suitable for denoising. ${ }^{48}$ Further, the cardiac pulsation (around $1 \mathrm{~Hz}$ ) may contain useful information for intraoperative pain assessment. ${ }^{43}$ In this study, the discrete stationary wavelet transform (DSWT) was employed for the decomposition of the optical signals, which performed a multilevel stationary wavelet decomposition using either a specific orthogonal wavelet or specific orthogonal wavelet decomposition filters. ${ }^{49,50}$ The inverse DSWT was used to reconstruct the signal at the proper scale. The details of the fNIRS data preprocessing are described as follows.

First, raw fNIRS data were decomposed into five subbands using the Daubechies-4 wavelet function based on the DSWT method. The DSWT decomposition schematic diagram of the optical signals is illustrated in Fig. 6. In implementing multiscale wavelet decomposition to the optical signals, the components of the approximation coefficient and detail coefficient at each level can be derived, as showed in Fig. 6 (left). Then, for the detail component of D3 and D2 containing the waveform range from 0.625 to $2.5 \mathrm{~Hz}$, which are mainly cardiac pulsation information, the CTHR index was extracted from these components with the windows of $1 \mathrm{~min}$ and $10 \mathrm{~s}$ overlap. Third, the hemodynamic parameters $\mathrm{HbO}_{2}$ and $\mathrm{Hb}$ oscillating at 0.01 to $0.15 \mathrm{~Hz}$ are believed to be closely related to brain activation. ${ }^{16}$ The A5 component $(<0.15 \mathrm{~Hz})$ was used to extract the relative concentration changes of $\mathrm{HbO}_{2}, \mathrm{Hb}$, and total hemoglobin $\left(\mathrm{HbT}, \mathrm{HbT}=\mathrm{HbO}_{2}+\mathrm{Hb}\right)$. The $10 \mathrm{~s}$ data prior to anesthesia induction were used as the baseline during the calculation of relative $\mathrm{HbO}_{2}$ and $\mathrm{Hb}$ (using MBLL). Finally, the motion correction method, based on empirical mode decomposition, proposed in our preview study was used to remove the motion artifacts. $^{51}$

\subsection{Statistical Analysis}

In order to analyze the change and distribution of the $\mathrm{HbO}_{2}$ and $\mathrm{Hb}$ during anesthesia, the distribution of the indices in the awake, induction, moderate anesthesia, and recovery states for all 11 subjects were analyzed. The Kolmogorov-Smirnov test (kstest.m) was used to compare the values of $\mathrm{HbO}_{2}$ and $\mathrm{Hb}$ in different states to a standard, normal distribution. The Box plot was employed to evaluate the performance of distinguishing different anesthetic states of $\mathrm{HbO}_{2}$ and $\mathrm{Hb}$ values. A multiple comparison test and Kruskal-Wallis test (kruskalwallis. $\mathrm{m})$ were used to determine the significant differences of the index values between awake and moderate anesthesia states and between moderate anesthesia and recovery states. ${ }^{52}$

\section{Results}

The cerebral hemodynamic changes of all 12 channels for one typical patient are shown in Fig. 7. The operation lasted for two

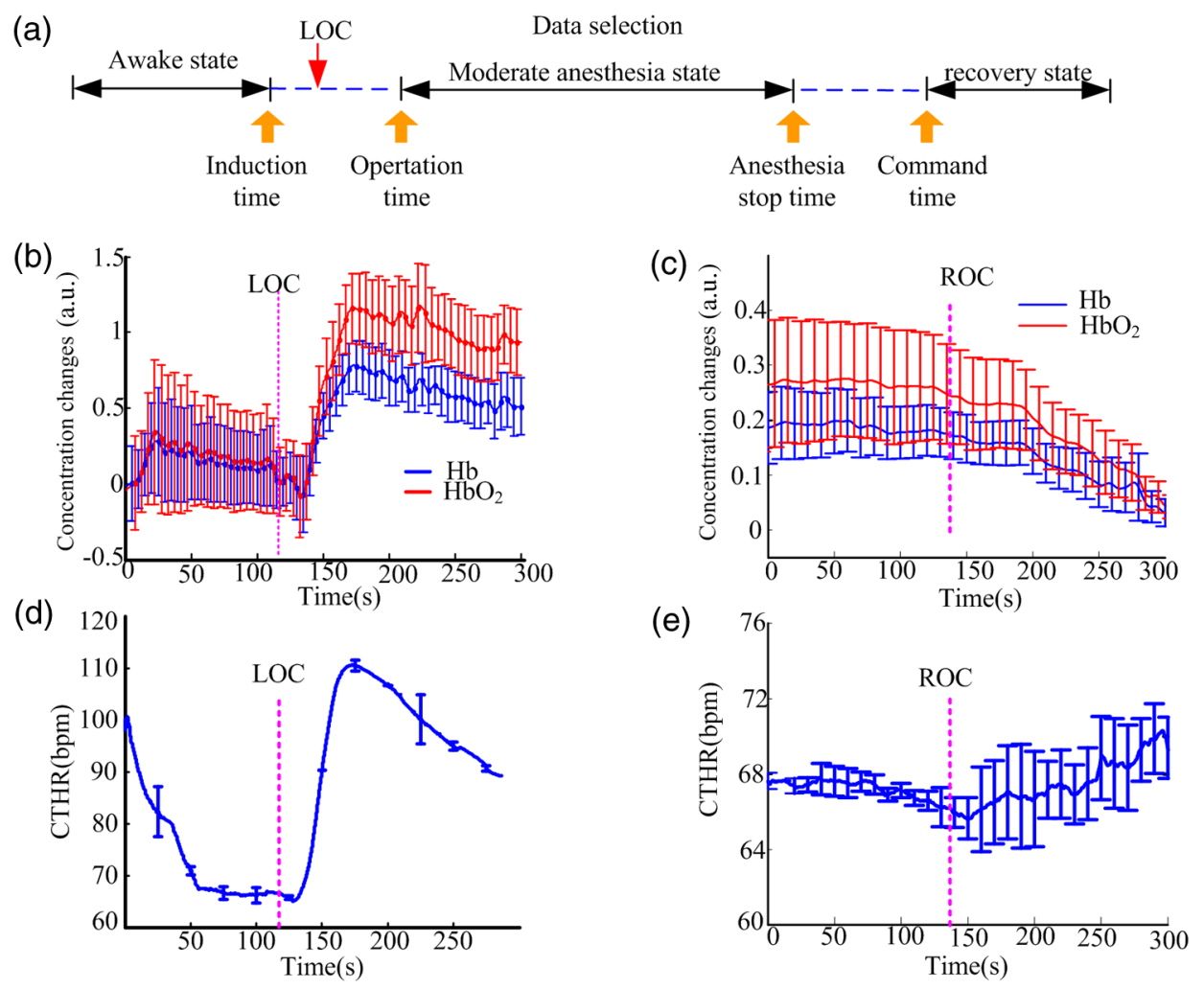

Fig. 8 The data recording process of fNIRS in surgery and the detailed changes of hemodynamic information and CTHR values during the induction and recovery of anesthesia from another patient. (a) The schematic diagram of the data recording process of the fNIRS with patients. (b) The $\mathrm{HbO}_{2}$ and $\mathrm{Hb}$ concentration changes during induction of anesthesia. The error bars are the standard deviation of indices with all channels at that time point, and the dashed vertical line indicates the time point of LOC. The data are selected about $300 \mathrm{~s}$ around the LOC. (c) Concentration changes of $\mathrm{HbO}_{2}$ and $\mathrm{Hb}$ during recovery of anesthesia. The dash vertical line indicates the time point of RoC. The data are selected about $300 \mathrm{~s}$ around the RoC. (d) and (e) The CTHR change during loss of anesthesia and recovery of anesthesia, respectively. 
and a half hours. The detailed anesthetic infusion process and the corresponding brain states are labeled with (I) to (VII) in Fig. 7(a). Figures 7(b)-7(d) show the concentration changes of $\mathrm{HbO}_{2}, \mathrm{Hb}$, and $\mathrm{HbT}$, respectively. The dark solid curve is the mean value of the cerebral hemodynamic indices, and the shaded area is the standard error during the whole course. It can be observed that $\mathrm{HbO}_{2}$ and $\mathrm{HbT}$ had changed during the whole anesthesia process.

In order to examine the performance of the fNIRS in detecting the effect of the anesthetic drug on the brain, the induction process (from the awake to the anesthetized state) and the emergence process (after the cessation of generation anesthetics) were analyzed. The time courses of LOC and recovery from another subject who underwent a laparoscopic prostatectomy were shown in Fig. 8. It can be seen that the relative $\mathrm{HbO}_{2}$ and $\mathrm{Hb}$ increased sharply after the LOC. Additionally, the indices of $\mathrm{HbO}_{2}$ and $\mathrm{Hb}$ decreased slowly during the emergency process. Also, the CTHR index changed in both the induction and awakening processes. The relative $\mathrm{HbO}_{2}$ fluctuation had a similar trend, ${ }^{28}$ while the trend is opposite in the case of $\mathrm{Hb}^{28}$ Interestingly, the $\mathrm{Hb}$ indices have similar trends in induction phase with another study, ${ }^{48}$ which used propofol induction.

Furthermore, in order to analyze the significance of relative $\mathrm{HbO}_{2}, \mathrm{Hb}, \mathrm{HbT}$, and CTHR in each anesthesia states we selected 30-s long hemodynamic signals at each state (awake, induction, moderate anesthesia, and recovery) in each patient (except the patient 4 and 10 in awake). The data sets of the awake state were selected before the "induction time," and the moderate anesthesia state data sets were selected between the "operation time" and "anesthesia stop time." The induction state was defined as the time range from LOC-100 $\mathrm{s}$ to LOC $+100 \mathrm{~s}$. The recovery state data were collected after the "command time" or in the recovery room. The box plot of relative $\mathrm{HbO}_{2}, \mathrm{Hb}, \mathrm{HbT}$ as well as the CTHR values at four different brain states (I awake, II induction, III moderate anesthesia, and IV recovery) are shown in Figs. 9(a)-9(d), respectively. The corresponding statistics were summarized in Table 2. The Kolmogorov-Smirnov test showed that the values of each hemodynamic parameter of each anesthesia state were
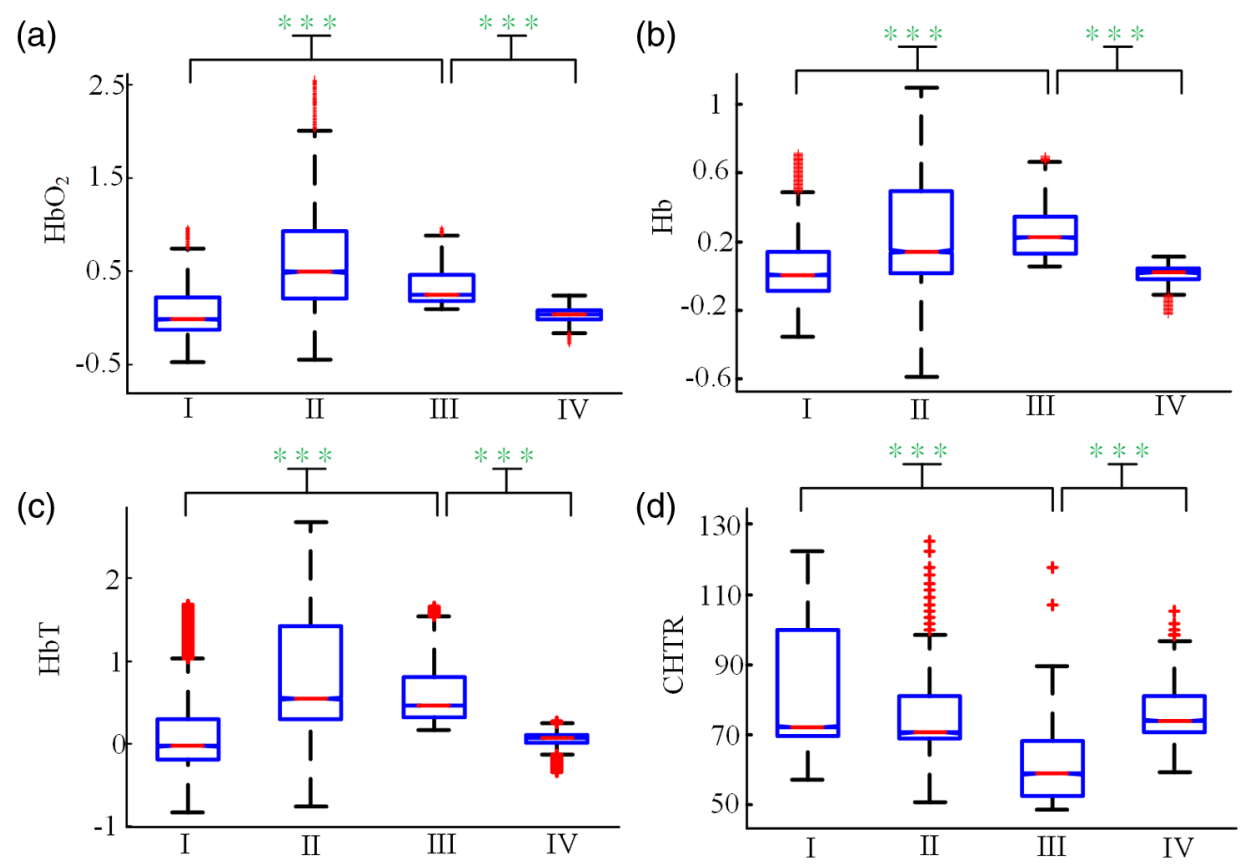

Fig. 9 Boxplot of $\mathrm{HbO}_{2}$ and $\mathrm{Hb}$ for the 11 patients at (I) awake, (II) induction, (III) moderate anesthesia, and (IV) recovery states. (a) and (b) The boxplot figures for $\mathrm{HbO}_{2}, \mathrm{Hb}, \mathrm{HbT}$, and CTHR at the four states, respectively. The notation ${ }^{*},{ }^{* *}$, and ${ }^{* \star *}$ indicate significant difference at $p<0.05, p<0.01$, and $p<0.001$, respectively (Kruskal-Wallis test and multiple comparison test).

Table 2 The statistics of the $\mathrm{HbO}_{2}, \mathrm{Hb}, \mathrm{HbT}$, and CTHR in the awake, induction, moderate anesthesia, and recovery states.

\begin{tabular}{|c|c|c|c|c|}
\hline & $\mathrm{HbO}_{2}$ & $\mathrm{Hb}$ & $\mathrm{HbT}$ & CTHR \\
\hline State & Median (min-max) & Median (min-max) & Median (min-max) & Median (min-max) \\
\hline Awake & $-0.016(-0.478$ to 0.968$)$ & $0.006(-0.354$ to 0.714$)$ & $-0.031(-0.832$ to 1.682$)$ & 72.29 (57.14 to 22.45$)$ \\
\hline Induction & $0.494(-0.449$ to 2.512$)$ & $0.143(-0.585$ to 1.093$)$ & $0.544(-0.759$ to 2.680$)$ & $70.59(50.86$ to 128.35$)$ \\
\hline Moderate anesthesia & $0.246(0.091$ to 0.961$)$ & $0.223(0.055$ to 0.695$)$ & $0.461(0.165$ to 1.657$)$ & $58.82(48.75$ to 118.32$)$ \\
\hline Recovery & $0.041(-0.198$ to 0.191$)$ & $0.019(-0.221$ to 0.112$)$ & $0.063(-0.344$ to 0.275$)$ & $74.07(59.41$ to 105.26$)$ \\
\hline
\end{tabular}


not normally distributed. The statistically significant difference among different brain states for each parameter is marked at the top of Fig. 9, using the notation *,**, and $* * *$ to indicate a significant difference at, $p<0.05, p<0.01$, and $p<0.001$, respectively (Kruskal-Wallis test and multiple comparison test). It can be seen that the concentration changes of $\mathrm{HbO}_{2}, \mathrm{Hb}, \mathrm{HbT}$, and CTHR are significantly different among the three states: awake, moderate, and recovery states. All the $p$-value are smaller than 0.001 in statistics.

\section{Discussion and Conclusion}

In this study, a new multichanneled fNIRS device based on frequency-division multiplexing principle was developed and applied in GA monitoring. The cerebral hemodynamic changes $\left(\mathrm{HbO}_{2}, \mathrm{Hb}\right.$, and $\left.\mathrm{HbT}\right)$ and CTHR were calculated, and the indices of each parameter at different anesthesia states were analyzed. The results showed that the $\mathrm{HbO}_{2}, \mathrm{Hb}$, and $\mathrm{HbT}$ derived from our equipment can be used to differentiate the brain states under propofol and sevoflurane anesthesia.

There are many commercially available fNIRS systems that have been used in brain function studies. Scholkmann et al. reviewed the CW-based fNIRS systems in detail ${ }^{24}$ and showed that there are more than 17 commercial fNIRS devices have been widely used in neuroscience research. However, the fNIRS system that can be used for diagnosis or monitoring patients during anesthesia is still limited. ${ }^{33}$ For example, clinically available fNIRS equipments, such as FORE-SIGHT and INVOS, are not able to elucidate the relationship between anesthesia and cognitive activity. Within the few NIRS studies on anesthesia, most of them focused on the effects of different anesthetic drugs on cerebral metabolism and hemodynamics. ${ }^{53,54}$ Hemandez-Meza et al. had used the NIRS to measure the prefrontal activity during the induction and recovery states of GA, as well as compare the $\mathrm{Hb}$ value with BIS in outpatient elective colonoscopy and coloproctology surgery. ${ }^{21,28,33}$ The results showed that the fNIRS have potential in assessing the DoA. However, there are still many problems.

In this study, we planned to validate the findings of other groups using the new fNIRS device. The design principle of the device is based on the principle of frequency-division multiplexing, which is widely adopted in the field, such as CW5, Braininsight NIRS, ETG-7100, and CW6. ${ }^{28,47}$ Compared to these existing commercial instruments, there are several advantages of our system:

i. Higher sampling rate $(100 \mathrm{kHz})$ and smaller size (using FPGA as the main processor). The parallel processing of FPGA guaranteed the sampling completely synchronized among channels.

ii. Each light source is driven by sinusoidal frequency modulation waves with the frequency ranging from 1 to $7 \mathrm{kHz}$, which prevents interference from the other light.

iii. The DSWT is adopted to extract $\mathrm{HbO}_{2}, \mathrm{Hb}, \mathrm{HbT}$, and CTHR. The demodulated signal is downsampled to $10 \mathrm{~Hz}$. It allows us to obtain more physiological information. The CTHR reflects the changes of the HR, which may have potential value in analgesia assessment. ${ }^{41}$

However, the cerebral hemodynamics could be affected by various factors, such as anesthetic drugs, surgical position, as well as the patient's physical conditions. ${ }^{21}$ In our study, all the patients underwent the peritoneoscope surgery and the results showed that the concentrations of $\mathrm{HbO}_{2}$ and $\mathrm{Hb}$ increased during induction state. In the work of LeonDominguez et al., ${ }^{48}$ it was found that $\mathrm{Hb}$ increased during the induction process under propofol and sevoflurane anesthesia. However, in another study, ${ }^{30}$ the $\mathrm{Hb}$ values slightly decreased or remained the same during induction under propofol sedation. From a neurophysiological viewpoint, during the anesthetic induction, when the patient's consciousness is diminishing, the PFC activity decreases, resulting in the increase of $\mathrm{Hb}^{33}$ The fact that $\mathrm{Hb}$ levels exhibited different trends in different channels may be explained by the different neural activation patterns in re-establishment of the high-order cognitive functions. ${ }^{55,56}$ In one review study, ${ }^{28}$ it was shown that the $\mathrm{HbT}$ increased during the propofol induction, which is consistent with our findings. Lastly, the change of $\mathrm{Hb}$ was selected for cortical activation analysis, ${ }^{48}$ due to its high temporal and spatial correlations with BOLD response, which has been demonstrated by concurrent fNIRS/fMRI studies. ${ }^{57}$

It is interesting that the four curves of CTHR, $\mathrm{Hb}, \mathrm{HbO}_{2}$, and HbT had the similar trends (seen in Fig. 7). The detailed changes of hemodynamic information and the CTHR values during the induction and recovery of anesthesia of another patient are shown in Fig. 8. It can be seen that the curves of $\mathrm{Hb}, \mathrm{HbO}_{2}$, and CTHR have a similar trend in the induction process. However, the $\mathrm{Hb}$ and $\mathrm{HbO}_{2}$ were not consistent with the CTHR in the ROC process. Further, the box plots of each index at awake, induction, moderate anesthesia, and recovery states are shown in Fig. 9. The statistics show that the CTHR in different states of anesthesia were not consistent with hemodynamic parameters. Although the four curves in Fig. 7 have similar trends, it is hard to say $\mathrm{Hb}, \mathrm{HbO}_{2}$, and $\mathrm{HbT}$ values are affected by CTHR. The research indicates that the increased heart-rate response may be caused by induction and intubation maneuvers. ${ }^{58}$ Also, it is known that the intubation requires tilting the head backward to insert the tube and this could cause the motion artifacts. This operation may confuse the influence of the intubation maneuvers and motion artifacts with the increase of $\mathrm{Hb}$ and $\mathrm{HbO}_{2}$. In addition, it is found that the decrease of the $\mathrm{Hb}$ may be caused by the decrease of HR and the drop of blood pressure after propofol perfusion. ${ }^{33}$ Unfortunately, the communication protocol is private and the commercial monitoring devices do not have a synchronous trigger port with other devices in this study, which made it impossible to compare the peripheral physiologies and hemodynamic parameters. In future studies, we will address these limitations.

Compared with hemodynamic parameters, the metabolic parameter of cerebral oxygen metabolism $\left(\mathrm{CMRO}_{2}\right)$ could provide critical diagnostic information for bedside measurement of brain health. ${ }^{59}$ It is well known that the $\mathrm{CMRO}_{2}$ could reflect neural hyperactivity and cell death. ${ }^{60}$ However, $\mathrm{CMRO}_{2}$ cannot be easily derived from a single functional brain imaging technique, such as fMRI, PET, and optical imaging. ${ }^{61}$ Especially, all the NIRS systems, which are based on the CW principle, could only obtain the hemodynamic parameters of relative $\mathrm{Hb}$ and $\mathrm{HbO}_{2}$. To obtain the $\mathrm{CMRO}_{2}$, additional optical imaging techniques are needed, such as diffuse correlation spectroscopy, to assess the value of regional cerebral blood flow. ${ }^{62}$

Furthermore, it is critical to quantify the effect of anesthetic on the brain in different concentrations. However, drug metabolism in the body is a complex nonlinear process. The pharmacokinetic-pharmacodynamic model was usually used to predict the effect on the brain. ${ }^{63}$ The anesthetic concentration effect can 
be significantly affected by the subject-specific physiological states. Moreover, more than one anesthetic drug was used in the study, different drugs may establish synergies with each other. In this study, we mainly focused on the fNIRS design and its effectiveness in evaluating GA through the comparison of hemodynamic parameters in different anesthesia states. In further studies, it is important to study in depth the internal relationship between metabolic process of anesthesia and hemodynamic effects on PFC, based on the pharmacokineticpharmacodynamic model.

Additionally, the $\mathrm{HbO}_{2}$ and $\mathrm{Hb}$ measurements can be affected by the hemodynamics of the superficial-layer. The multidistance measurement should be considered in the future to remove the noise from the superficial layer. ${ }^{64}$ Meanwhile, the spatial information of hemodynamic changes is also important to reflect the effect of anesthesia. One study ${ }^{33}$ showed that the $\mathrm{Hb}$ value increased during the induction state and had different trends during the emergency state in different channels. In this study, we analyzed the spatial fluctuations in $\mathrm{HbO}_{2}$ and $\mathrm{Hb}$ with changes of anesthesia in different anesthesia states. However, we did not find significant correlations. There may be several reasons, such as differences in anesthetic drugs, surgery positions, and the patient's physical conditions. ${ }^{21}$ It is also suggested that the apparent disorganization of hemodynamic changes in fNIRS channels in the emergence phase may relate to the reestablishment of high-order cognitive functions. ${ }^{55,56}$ The spatial relationship of consciousness and hemodynamic changes requires more carefully designed studies.

In conclusion, we presented a multichanneled fNIRS system and proved its effectiveness in DoA monitoring. However, our study merely considered the temporal cerebral hemodynamic information at different anesthesia states. More studies are needed to understand the spatial hemodynamic information. Furthermore, to become a useful DoA monitor, the index quantification, and the internal relations with EEG-based DoA monitor should be established.

\section{Acknowledgments}

This research was supported by National Natural Science Foundation of China $(61304247,81230023,61273063)$, China Postdoctoral Science Foundation (2014M551051, 2015T80231), Natural Science Foundation of Heibei Province of China (F2014203127), and the Education Department of Hebei province (GCC2014019). The commercialization of the research fund is supported by Beijing Municipal Commission of Education. The authors declare that no conflicts of interest exist.

\section{References}

1. L. D. Lewis et al., "Rapid fragmentation of neuronal networks at the onset of propofol-induced unconsciousness," Proc. Natl. Acad. Sci. U. S. A. 109(49), E3377-E3386 (2012).

2. S. Newman et al., "Postoperative cognitive dysfunction after noncardiac surgery: a systematic review," Anesthesiology 106(3), 572-590 (2007).

3. S. Ching and E. N. Brown, "Modeling the dynamical effects of anesthesia on brain circuits," Curr. Opin. Neurobiol. 25, 116-122 (2014).

4. P. L. Purdon et al., "Electroencephalogram signatures of loss and recovery of consciousness from propofol," Proc. Natl. Acad. Sci. U. S. A. 110(12), E1142-E1151 (2013).

5. J. Bruhn et al., "Depth of anaesthesia monitoring: what's available, what's validated and what's next?" Br. J. Anaesth. 97(1), 85-94 (2006).

6. H. Viertiö-Oja et al., "Description of the entropy ${ }^{\mathrm{TM}}$ algorithm as applied in the Datex-Ohmeda S $/ 5^{\mathrm{TM}}$ entropy module," Acta Anaesthesiol. Scand. 48(2), 154-161 (2004).
7. P. Myles, J. Symons, and K. Leslie, “Anaesthetists' attitudes towards awareness and depth-of-anaesthesia monitoring," Anaesthesia 58(1), 11-16 (2003).

8. I. J. Rampil, "A primer for EEG signal processing in anesthesia," J. Am. Soc. Anesthesiol. 89, 980-1002 (1998).

9. M. Murphy et al., "Propofol anesthesia and sleep: a high-density EEG study," Sleep 34(3), 283-291A (2011).

10. M. S. Avidan and G. A. Mashour, "The incidence of intra-operative awareness in the UK: under the rate or under the radar?" $B r . J$. Anaesth. 110(4), 494-497 (2013).

11. S. C. Manyam et al., "When is a bispectral index of 60 too low? Rational processed electroencephalographic targets are dependent on the sedative-opioid ratio," Anesthesiology 106(3), 472-483 (2007).

12. S. V. Delius et al., "Auditory evoked potentials compared with bispectral index for monitoring of midazolam and propofol sedation during colonoscopy," Am. J. Gastroenterol. 104(2), 318-325 (2009).

13. G. S. Murphy et al., "Cerebral oxygen desaturation events assessed by near-infrared spectroscopy during shoulder arthroscopy in the beach chair and lateral decubitus positions," Anesth. Analg. 111(2), 496-505 (2010).

14. S. Tan, "Cerebral oximetry in cardiac surgery," Hong Kong Med. J. 14(3), 220-225 (2008).

15. A. Villringer and U. Dirnagl, "Coupling of brain activity and cerebral blood flow: basis of functional neuroimaging," Cerebrovasc. Brain Metab. Rev. 7(3), 240-276 (1995).

16. Y. Tong, L. M. Hocke, and S. C. Licata, "Low-frequency oscillations measured in the periphery with near-infrared spectroscopy are strongly correlated with blood oxygen level-dependent functional magnetic resonance imaging signals," J. Biomed. Opt. 17(10), 106004 (2012).

17. S. Tak and J. C. Ye, "Statistical analysis of fNIRS data: a comprehensive review," Neuroimage 85, 72-91 (2014).

18. K. Izzetoglu, "Neural correlates of cognitive workload and anesthetic depth: fNIR spectroscopy investigation in humans," Drexel University (2008).

19. S. M. Coyle, T. E. Ward, and C. M. Markham, "Brain-computer interface using a simplified functional near-infrared spectroscopy system," J. Neural Eng. 4(3), 219 (2007).

20. M. Ferrari and V. Quaresima, "A brief review on the history of human functional near-infrared spectroscopy (fNIRS) development and fields of application," Neuroimage 63(2), 921-935 (2012).

21. A. Curtin et al., "Functional near-infrared spectroscopy for the measurement of propofol effects in conscious sedation during outpatient elective colonoscopy," Neuroimage 85, 626-636 (2014).

22. M. Lipcsey, N. C. Woinarski, and R. Bellomo, "Near infrared spectroscopy (NIRS) of the thenar eminence in anesthesia and intensive care," Ann. Intensive Care 2(1), 11 (2012).

23. V. Quaresima, S. Bisconti, and M. Ferrari, "A brief review on the use of functional near-infrared spectroscopy (fNIRS) for language imaging studies in human newborns and adults," Brain Lang. 121(2), 79-89 (2012).

24. F. Scholkmann et al., "A review on continuous wave functional nearinfrared spectroscopy and imaging instrumentation and methodology," Neuroimage 85, 6-27 (2014).

25. T. Muehlemann, D. Haensse, and M. Wolf, "Wireless miniaturized invivo near infrared imaging," Opt. Express 16(14), 10323-10330 (2008).

26. A. Torricelli et al., "Time domain functional NIRS imaging for human brain mapping," Neuroimage 85(Pt 1), 28-50 (2014).

27. D. Ramsingh, "Brain oxygenation," in Monitoring Technologies in Acute Care Environments: A Comprehensive Guide to Patient Monitoring Technology, M. J. Ehrenfeld and M. Cannesson, Eds., pp 241-245, Springer, New York, New York (2014).

28. G. Hernandez-Meza et al., "Near-infrared spectroscopy for the evaluation of anesthetic depth," Biomed. Res. Int. 2015, 939418 (2015).

29. S. Shidoh et al., "The process of change in hemodynamics after revascularization in the ischemic brain," NeuroReport 26(11), 629-633 (2015).

30. A. Curtin et al., "Functional near-infrared spectroscopy for the measurement of propofol effects in conscious sedation during outpatient elective colonoscopy," Neuroimage 85(Pt 1), 626-636 (2014).

31. A. T. Lovell et al., "Continuous measurement of cerebral oxygenation by near infrared spectroscopy during induction of anesthesia," Anesth. Analg. 88(3), 554-558 (1999). 
32. A. Fassoulaki et al., "The effect of desflurane and sevoflurane on cerebral oximetry under steady-state conditions," Anesth. Analg. 102(6), 1830-1835 (2006).

33. U. Leon-Dominguez et al., "Molecular concentration of deoxyHb in human prefrontal cortex predicts the emergence and suppression of consciousness," Neuroimage 85, 616-625 (2014).

34. S.-J. Kim et al., "The effects of sevoflurane and propofol anesthesia on cerebral oxygenation in gynecological laparoscopic surgery," Korean J. Anesthesiol. 61(3), 225-232 (2011).

35. S. Pant, D. J. Bokor, and A. K. Low, "Cerebral oxygenation using nearinfrared spectroscopy in the beach-chair position during shoulder arthroscopy under general anesthesia,' Arthroscopy 30(11), 15201527 (2014).

36. M. A. Franceschini et al., "Diffuse optical imaging of the whole head," J. Biomed Opt. 11(5), 054007 (2006).

37. H. Obrig et al., "Spontaneous low frequency oscillations of cerebral hemodynamics and metabolism in human adults," Neuroimage 12(6), 623-639 (2000).

38. V. Toronov et al., "Near-infrared study of fluctuations in cerebral hemodynamics during rest and motor stimulation: temporal analysis and spatial mapping," Med. Phys. 27(4), 801-815 (2000).

39. S. G. Diamond et al., "Dynamic physiological modeling for functional diffuse optical tomography," Neuroimage 30(1), 88-101 (2006).

40. K. von Siebenthal et al., "Cyclical fluctuations in blood pressure, heart rate and cerebral blood volume in preterm infants," Brain Dev. 21(8), 529-534 (1999).

41. T. M. Hemmerling et al., "The 'Analgoscore': a novel score to monitor intraoperative pain and its use for remifentanil closed-loop application," in IEEE Int. Conf. on Systems, Man and Cybernetics (2007).

42. T. M. Hemmerling et al., "Evaluation of a novel closed-loop total intravenous anaesthesia drug delivery system: a randomized controlled trial," Br. J. Anaesth. 110(6), 1031-1039 (2013).

43. Y. Tong, K. P. Lindsey, and B. deB Frederick, "Partitioning of physiological noise signals in the brain with concurrent near-infrared spectroscopy and fMRI," J. Cereb. Blood Flow Metab. 31(12), 2352-2362 (2011).

44. K. L. Perdue et al., "Extraction of heart rate from functional near-infrared spectroscopy in infants," J. Biomed. Opt. 19(19), 067010 (2014).

45. C. H. Schmitz et al., "Instrumentation for fast functional optical tomography," Rev. Sci. Instrum. 73(2), 429-439 (2002).

46. W. V. Drongelen, "Signal processing for neuroscientists," Signal Process. Neuroscientists 233(4764), 177-178 (2007).

47. G. $\mathrm{Xu}$ et al., "A DAQ-device-based continuous wave near-infrared spectroscopy system for measuring human functional brain activity," Comput. Math. Methods Med. 2014(3), 107320 (2014).

48. U. Leon-Dominguez et al., "Molecular concentration of deoxyHb in human prefrontal cortex predicts the emergence and suppression of consciousness," Neuroimage 85(Pt 1), 616-625 (2014).

49. N. Jmail et al., "A comparison of methods for separation of transient and oscillatory signals in EEG," J. Neurosci. Methods 199(2), 273-289 (2011).

50. M. R. Mowla et al., "Artifacts-matched blind source separation and wavelet transform for multichannel EEG denoising," Biomed. Signal Process. Control 22, 111-118 (2015).

51. Y. Gu et al., "Empirical mode decomposition-based motion artifact correction method for functional near-infrared spectroscopy," J. Biomed. Opt. 21(1), 015002 (2016).

52. Z. Liang et al., "A comparison of different synchronization measures in electroencephalogram during propofol anesthesia," Int. J. Clin. Monit. Comput. 30(4), 451-466 (2015).

53. K. Iwasaki et al., "Vital capacity induction with $8 \%$ sevoflurane and N2o causes cerebral hyperemia," J. Anesth. 17(1), 3-7 (2003).
54. R. A. De Blasi et al., "Effects of remifentanil-based general anaesthesia with propofol or sevoflurane on muscle microcirculation as assessed by near-infrared spectroscopy," Br. J. Anaesth. 101(2), 171-177 (2008).

55. J. Leon-Carrion et al., "Differential time course and intensity of PFC activation for men and women in response to emotional stimuli: a functional near-infrared spectroscopy (fNIRS) study," Neurosci. Lett. 403(1-2), 90-95 (2006).

56. J. Leoncarrion et al., "Time-locked brain activity associated with emotion: a pilot MEG study," Brain Inj. 20(8), 857-865 (2006).

57. T. J. Huppert et al., "A temporal comparison of BOLD, ASL, and NIRS hemodynamic responses to motor stimuli in adult humans," NeuroImage 29(2), 368-382 (2006).

58. E. N. Brown, R. Lydic, and N. D. Schiff, "General anesthesia, sleep, and coma," N. Engl. J. Med. 363(27), 2638-2650 (2010).

59. D. W. Brown, J. Hadway, and T. Y. Lee, "Near-infrared spectroscopy measurement of oxygen extraction fraction and cerebral metabolic rate of oxygen in newborn piglets," Pediatr. Res. 54(6), 861-867 (2003).

60. P. Y. Lin et al., "Non-invasive optical measurement of cerebral metabolism and hemodynamics in infants," J. Visualized Exp. 73, e4379 (2013).

61. A. K. Dunn et al., "Spatial extent of oxygen metabolism and hemodynamic changes during functional activation of the rat somatosensory cortex," Neurolmage 27(2), 279-290 (2005).

62. P. L. Purdon et al., "Systems and methods for monitoring brain metabolism and activity using electroencephalogram and optical imaging," (2014).

63. Z. Liang et al., "EEG entropy measures in anesthesia," Front. Comput. Neurosci. 9, 16 (2015).

64. M. Dehaes et al., "Assessment of the frequency-domain multi-distance method to evaluate the brain optical properties: Monte Carlo simulations from neonate to adult," Biomed. Opt. Express 2(3), 552-567 (2011).

Zhenhu Liang is an associate professor in the Department of Automation at Yanshan University. He received his BEng degree in mechanical engineering and his PhD in automation from Yanshan University, China, in 2004 and 2012, respectively. His current research interests include neural engineering, signal processing, data analysis, and monitoring system design.

Yue Gu received his BSc and MEng degrees in automation from Yanshan University, Qinhuangdao, China. He is currently working towards his PhD at the School of Electrical Engineering, Yanshan University. His research interests include fNIRS signal processing and application of fNIRS in brain development of children.

Lei Cheng received his BSc degree in electrical engineering and automation from Yanshan University, Qinhuangdao, China. He is currently working towards his MEng at the School of Electrical Engineering, Yanshan University. His research interests include fNIRS signal processing and system design.

Xiaoli Li received his $\mathrm{BE}$ and $\mathrm{ME}$ degrees in mechanical engineering from Kunming University of Science and Technology and his PhD from Harbin Institute of Technology, China. He is currently a professor and vice director with the National Key Laboratory of Cognitive Neuroscience and Learning, Beijing Normal University, China. His research interests include neural engineering, computational intelligence, signal processing and data analysis, monitoring system, and manufacturing system.

Biographies for the other authors are not available. 